\title{
Association of G2350A polymorphism of angiotensin converting enzyme gene with essential hypertension and type 2 diabetes mellitus in Malaysian subjects.
}

\begin{abstract}
The objective of this study was to determine the association of Angiotensin-Converting Enzyme (ACE) G2350A polymorphism in Essential Hypertension (EHT) and Type 2 Diabetes Mellitus (T2DM) in Malaysian subjects. A total of 265 samples have been recruited for this study. In order to amplify the 122 bp of G2350A variant of ACE gene, genomic DNA was amplified using Hot-start Polymerase-Chain Reaction (PCR) and the PCR products was digested with BstuI restriction enzyme to produce 103 and $19 \mathrm{bp}$ when $\mathrm{A}$ is at position 2350. The subjects were ranged from 30 to 84 years old witi mean age of 51.84 years. The frequency for GG, GA and AA genotypes of ACE gene was 41.43, 48.57 and $10 \%$ in EHT, 46.67, 36.67 and $16.66 \%$ in T2DM, 32.31, 53.85 and 13.84\% in T2DM with EHT compared to $61.43,35.71$ and $2.86 \%$ of control subjects respectively. The frequency for A allele of G2350A polymorphism was $34.29 \%$ in EHT, $35.00 \%$ in T2DM, $40.77 \%$ in T2DM+EHT subjects compared to controls (20.71\%). The genotype and allele frequency of ACE G2350A gene polymorphism differed significantly in patients when compared to controls $(p<0.05)$. Allele A of G2350A polymorphism of ACE gene is associated with essential hypertension and Type 2 Diabetes Mellitus in Malaysian subjects.
\end{abstract}

Keyword: ACE engiotensin-converting enzyme gene; Essential hypertension; G2350A polymorphism; Type 2 diabetes mellitus 